\title{
Sum Power Minimization for Cellular Systems With Underlay D2D Communications
}

\author{
Amin Ghazanfari, Antti Tölli and Harri Pennanen \\ Centre for Wireless Communications, University of Oulu \\ P.O. Box 4500, 90014 University of Oulu, Finland \\ Email: aghazanf@ee.oulu.fi, antti.tolli@ee.oulu.fi and harri.pennanen@ee.oulu.fi
}

\begin{abstract}
In this paper, we consider a single-cell system where the same radio resources are simultaneously used by a cellular user and a pair of device-to-device (D2D) terminals. The optimization objective is to minimize the sum transmission power of the system while satisfying the user specific minimum rate constraints. We propose joint power control and beamforming algorithms to solve the power minimization problem optimally both in uplink and downlink. In the uplink, the optimal transmit powers for the cellular and D2D users are obtained via fixedpoint iterations, whereas the linear minimum mean squared error receiver is used for optimal reception at the base station (BS). In the downlink, the problem is equivalently reformulated as a second-order cone program (SOCP). As a result, the optimal transmit beamformer for the cellular user and the optimal transmit power for the D2D user can be efficiently computed via standard SOCP solvers. Simulation results demonstrate that the sum power performance can be significantly improved as compared with the conventional cellular system. Results also illustrate that the power consumption of the network is highly affected by the locations of the cellular and D2D users, and whether the resources are shared in the uplink or downlink.
\end{abstract}

\section{INTRODUCTION}

Device-to-device (D2D) communications amongst cellular users that are in the vicinity of each other is introduced as a promising approach to provide high data rate, low latency and low power communication [1]. These benefits are mainly due to short distance and direct communication between D2D users. In addition, it offers data offloading and improves cell coverage [1]-[3]. To summarize, D2D communication can improve the spectral and energy efficiency of cellular networks. Thus, the idea of network assisted D2D communications underlaying cellular networks has recently attracted a lot of attention in wireless communication research community, see for example [1]-[3], and the references therein. Nevertheless, it also introduces new challenges for cellular networks, i.e., extra interference is generated between D2D and cellular communication that are sharing the same resources [1]. Mode selection, power control and scheduling are proposed as efficient techniques to control the generated interference towards the cellular system [1].

Power control in cellular network with an underlaying D2D connection was considered in [4] to manage the extra interference. In [5], a novel power control mechanism was

This work has been supported by the Finnish Funding Agency for Technology and Innovation (Tekes), Nokia, Huawei Technologies Co. Ltd. and Broadcom Corporation. presented which exploits two new methods for power control (i.e., D2D backoff and cellular boosting). These two methods were used to decrease interference by controlling the transmit power of D2D transmitter. There are some works that define proper area for D2D communications according to the fixed interference to signal ratio, e.g., in [6] the area is called interference limited area which let users communicate in D2D mode if there is not any active cellular user. In [7], the authors proposed a heuristic interference management approach for the downlink resource sharing in D2D underlaying cellular networks. In order to mitigate the interference and improve the throughput, two different interference limited areas were defined for the D2D transmitter and receiver. Most of the previous works in the literature consider only single antenna cases with power control either in uplink or downlink phase.

In this paper, we propose sum power optimization schemes with joint power control and beamforming mechanisms for a cellular system with underlaying D2D communications in uplink and downlink. We consider a single-cell system which consists of a multi-antenna base station (BS) communicating with a cellular user, and an underlaying D2D pair, which exploits the same radio resources. The optimization objective is to minimize the total transmission power of the system while satisfying the predefined per user rate targets. In the uplink resource sharing case, a joint uplink power control and receive beamforming algorithm is proposed to solve the power minimization problem. The optimal uplink powers for the users are obtained via fixed-point iterations, whereas the optimal receive beamforming at the BS is achieved by using the linear minimum mean square error (MMSE) receiver. In the downlink resource sharing case, a centralized algorithm is proposed to solve the sum power optimization problem by reformulating it as a second-order cone program (SOCP), and solving it efficiently via standard SOCP solvers. As a result, the optimal transmit power for the D2D user and the optimal transmit beamformer for the cellular user are obtained. The proposed algorithms are controlled by the BS, and thus, it is required that the BS has the knowledge of all the channels in the system. The performance of the proposed algorithms is compared with the conventional cellular case via numerical examples. The paper is organized as follows. The system model, problem formulation and proposed algorithms are described in Sections II, III and IV, respectively. Sections V and VI present numerical results and conclusion, respectively. 


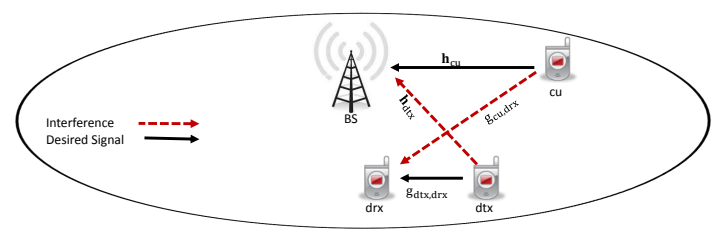

(a) Uplink communication.

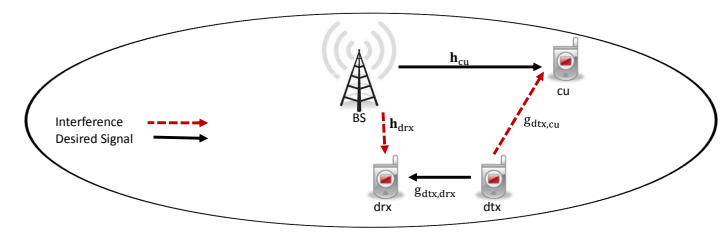

(b) Downlink communication.

Fig. 1. Uplink and downlink communication scenarios with underlaying D2D pair.

\section{System ModeL}

Let us consider a single-cell network with a cellular user and a single D2D pair. In this system, each user is equipped with a single antenna, whereas the BS has $T$ antennas. The $\mathrm{BS}$ is in charge of controlling the cellular user and the underlay D2D communication. Furthermore, the cellular user and D2D pair have equal priority in the system. D2D underlay communication is considered in both uplink and downlink. These systems are illustrated in Figs. 1(a) and 1(b). We denote the cellular user, D2D transmitter and D2D receiver by cu, dtx and drx, respectively.

In the uplink phase, the received signal vector $\mathbf{y}_{\mathrm{bs}}$ at the $\mathrm{BS}$ is given by

$$
\mathbf{y}_{\mathrm{bs}}=\sqrt{p_{\mathrm{cu}}} \mathbf{h}_{\mathrm{cu}}^{H} d_{\mathrm{cu}}+\sqrt{p_{\mathrm{dtx}}} \mathbf{h}_{\mathrm{dtx}}^{H} d_{\mathrm{dtx}}+\mathbf{n}_{\mathrm{bs}}
$$

where $p_{\text {cu }}$ is the uplink transmit power of the cellular user, $\mathbf{h}_{\text {cu }} \in \mathbb{C}^{1 \times T}$ is the channel vector between the cellular user and the BS and $d_{\mathrm{cu}}$ is the data symbol for the cellular user. Similarly, $p_{\mathrm{dtx}}$ is the transmitted power of the D2D transmitter, $\mathbf{h}_{\mathrm{dtx}}$ is the channel vector from the D2D transmitter to the BS and $d_{\mathrm{dtx}}$ is the data symbol transmitted from the D2D transmitter. The vector $\mathbf{n}_{\mathrm{bs}}$ indicates the additive white Gaussian noise with zero mean and variance $N_{0}$ per element. The estimated data symbol $\hat{d}_{\mathrm{cu}}$ of the cellular user at the BS after applying receive beamforming is expressed as

$$
\hat{d}_{\mathrm{cu}}=\mathbf{w}_{\mathrm{cu}}^{H} \mathbf{y}_{\mathrm{bs}}
$$

where $\mathbf{w}_{\mathrm{cu}} \in \mathbb{C}^{T}$ is the uplink receive beamforming vector for the cellular user. The received signal $y_{\mathrm{drx}}$ at the $\mathrm{D} 2 \mathrm{D}$ receiver in the uplink phase is written as

$$
y_{\mathrm{drx}}=\sqrt{p_{\mathrm{dtx}}} g_{\mathrm{dtx}, \mathrm{drx}} d_{\mathrm{dtx}}+\sqrt{p_{\mathrm{cu}}} g_{\mathrm{cu}, \mathrm{drx}} d_{\mathrm{cu}}+n_{\mathrm{drx}}
$$

where $g_{\mathrm{dtx}, \mathrm{drx}} \in \mathbb{C}$ is the channel response between the D2D transmitter and the D2D receiver, $g_{\mathrm{cu}, \mathrm{drx}}$ is the channel from the cellular user to the $\mathrm{D} 2 \mathrm{D}$ receiver and $n_{\mathrm{drx}}$ is the additive white Gaussian noise sample with zero mean and variance $N_{0}$.

In the downlink phase, the received signal $\tilde{y}_{\mathrm{cu}}$ at the cellular user can be written as

$$
\tilde{y}_{\mathrm{cu}}=\mathbf{h}_{\mathrm{cu}} \mathbf{m}_{\mathrm{cu}} d_{\mathrm{cu}}+\sqrt{\tilde{p}_{\mathrm{dtx}}} g_{\mathrm{dtx}, \mathrm{cu}} d_{\mathrm{dtx}}+n_{\mathrm{cu}}
$$

where $\mathbf{m}_{\mathrm{cu}} \in \mathbb{C}^{T}$ is the downlink transmit beamforming vector for the cellular user, $d_{\mathrm{cu}}$ is the data symbol for the cellular user, $\tilde{p}_{\mathrm{dtx}}$ is the power of the D2D transmitter, $g_{\mathrm{dtx}, \mathrm{cu}}$ is the channel from the D2D transmitter to the cellular user and $n_{\mathrm{cu}}$ is the white Gaussian noise sample for the cellular user.
The received signal $\tilde{y}_{\mathrm{drx}}$ at the D2D receiver in the downlink phase is expressed as

$$
\tilde{y}_{\mathrm{drx}}=\sqrt{\tilde{p}_{\mathrm{dtx}}} g_{\mathrm{dtx}, \mathrm{drx}} d_{\mathrm{dtx}}+\mathbf{h}_{\mathrm{drx}} \mathbf{m}_{\mathrm{cu}} d_{\mathrm{cu}}+n_{\mathrm{drx}}
$$

where $\mathbf{h}_{\mathrm{drx}}$ is the channel vector from the BS to the D2D receiver.

\section{PROBlEM Formulation}

In this work, the optimization objective is to minimize the sum transmission power of the whole system while the user specific rate targets are satisfied. It is assumed that both cellular and D2D links have equal rate targets for the uplink and downlink communications. Consequently, the optimization problem can be separated between the uplink and downlink. The resulting optimization problem for the uplink phase can be written as

$$
\begin{array}{ll}
\underset{p_{\mathrm{cu}}, p_{\mathrm{dtx}}, \mathbf{w}_{\mathrm{cu}}}{\min } & p_{\mathrm{cu}}+p_{\mathrm{dtx}} \\
\text { s. t. } & \log _{2}\left(1+\frac{p_{\mathrm{cu}}\left|\mathbf{w}_{\mathrm{cu}}^{H} \mathbf{h}_{\mathrm{cu}}^{H}\right|^{2}}{p_{\mathrm{dtx}}\left|\mathbf{w}_{\mathrm{cu}}^{H} \mathbf{h}_{\mathrm{dtx}}^{H}\right|^{2}+N_{0}}\right) \geq R_{\mathrm{cu}} \\
& \log _{2}\left(1+\frac{p_{\mathrm{dtx}}\left|g_{\mathrm{dtx}, \mathrm{drx}}\right|^{2}}{p_{\mathrm{cu}}\left|g_{\mathrm{cu}, \mathrm{drx}}\right|^{2}+N_{0}}\right) \geq R_{\mathrm{drx}}
\end{array}
$$

Note that the receive beamforming vector is normalized, i.e., $\left\|\mathbf{w}_{\mathrm{cu}}\right\|_{2}=1$. The fixed values $R_{\mathrm{cu}}$ and $R_{\mathrm{drx}}$ are the minimum rate targets for the cellular and D2D users, respectively. Note that the rate constraints in (6) can be equivalently mapped into the signal-to-interference-plus-noise ratio (SINR) constraints. The resulting equivalent uplink optimization problem is given by

$$
\begin{array}{ll}
\underset{p_{\mathrm{cu}}, p_{\mathrm{dtx}}, \mathbf{w}_{\mathrm{cu}}}{\min } & p_{\mathrm{cu}}+p_{\mathrm{dtx}} \\
\text { s. t. } & \frac{p_{\mathrm{cu}}\left|\mathbf{w}_{\mathrm{cu}}^{H} \mathbf{h}_{\mathrm{cu}}^{H}\right|^{2}}{p_{\mathrm{dtx}}\left|\mathbf{w}_{\mathrm{cu}}^{H} \mathbf{h}_{\mathrm{dtx}}^{H}\right|^{2}+N_{0}} \geq \gamma_{\mathrm{cu}} \\
& \frac{p_{\mathrm{dtx}}\left|g_{\mathrm{dtx}, \mathrm{drx}}\right|^{2}}{p_{\mathrm{cu}}\left|g_{\mathrm{cu}, \mathrm{drx}}\right|^{2}+N_{0}} \geq \gamma_{\mathrm{drx}}
\end{array}
$$

where $\gamma_{\mathrm{cu}}=2^{R_{\mathrm{cu}}}-1$ and $\gamma_{\mathrm{drx}}=2^{R_{\mathrm{drx}}}-1$ are the fixed SINR targets. Similarly, the optimization problem for the downlink communication phase is formulated as

$$
\begin{aligned}
& \underset{\tilde{p}_{\mathrm{dtx}}, \mathbf{m}_{\mathrm{cu}}}{\min }\left\|\mathbf{m}_{\mathrm{cu}}\right\|_{2}^{2}+\tilde{p}_{\mathrm{dtx}} \\
& \text { s. t. } \quad \frac{\left|\mathbf{h}_{\mathrm{cu}} \mathbf{m}_{\mathrm{cu}}\right|^{2}}{\tilde{p}_{\mathrm{dtx}}\left|g_{\mathrm{dtx}, \mathrm{cu}}\right|^{2}+N_{0}} \geq \gamma_{\mathrm{cu}} \\
& \frac{\tilde{p}_{\mathrm{dtx}}\left|g_{\mathrm{dtx}, \mathrm{drx}}\right|^{2}}{\left|\mathbf{h}_{\mathrm{drx}} \mathbf{m}_{\mathrm{cu}}\right|^{2}+N_{0}} \geq \gamma_{\mathrm{drx}} \text {. }
\end{aligned}
$$




\section{Proposed Algorithms}

\section{A. Uplink Communications}

In this section, we propose a joint power control and receive beamforming algorithm to optimally solve the uplink sum power minimization problem (7). The proposed algorithm is derived by setting the Lagrangian of (7) to zero (w.r.t. $\mathbf{w}_{\mathrm{cu}}$ ) and then performing some rearranging, similar to that in [8]. After these steps, the optimal powers of the cellular user and D2D transmitter can be iteratively calculated via the following fixed-point iterations

$$
\begin{gathered}
p_{\mathrm{cu}}[t+1]=\frac{\gamma_{\mathrm{cu}}}{\mathbf{h}_{\mathrm{cu}}\left(N_{0} \mathbf{I}+p_{\mathrm{dtx}}[t] \mathbf{h}_{\mathrm{dtx}}^{H} \mathbf{h}_{\mathrm{dtx}}\right)^{-1} \mathbf{h}_{\mathrm{cu}}^{H}} \\
p_{\mathrm{dtx}}[t+1]=\frac{\gamma_{\mathrm{drx}}}{g_{\mathrm{dtx}, \mathrm{drx}}\left(N_{0}+p_{\mathrm{cu}}[t] g_{\mathrm{cu}, \mathrm{drx}}^{H} g_{\mathrm{cu}, \mathrm{drx}}\right)^{-1} g_{\mathrm{dtx}, \mathrm{drx}}^{H}} .
\end{gathered}
$$

Following the proof in [8], it can be shown that (9) and (10) are standard functions, which converge to the optimal solutions. The optimal receive beamformer at the BS can be calculated by using the (scaled) linear MMSE receiver [9]:

$$
\mathbf{w}_{\mathrm{cu}}=\frac{\hat{\mathbf{w}}_{\mathrm{cu}}}{\left\|\hat{\mathbf{w}}_{\mathrm{cu}}\right\|_{2}}, \quad \hat{\mathbf{w}}_{\mathrm{cu}}=\left(N_{0} \mathbf{I}+p_{\mathrm{dtx}} \mathbf{h}_{\mathrm{dtx}}^{H} \mathbf{h}_{\mathrm{dtx}}\right)^{-1} \mathbf{h}_{\mathrm{cu}}^{H} \text {. }
$$

The proposed uplink power control and receive beamforming approach is summarized in Algorithm 1.

\begin{tabular}{l} 
Algorithm 1 Uplink power control and receive beamforming \\
\hline 1: Set $t=0$. Initialize $p_{\mathrm{cu}}(0)$ and $p_{\mathrm{dtx}}(0)$ \\
2: Repeat \\
3: Calculate the transmit powers $p_{\mathrm{cu}}(t+1)$ and $p_{\mathrm{dtx}}(t+1)$ \\
using (9) and (10), respectively. Set $t=t+1$ \\
4: Until stopping criterion is satisfied. \\
5: Calculate the receive beamformer $\mathbf{w}_{\mathrm{cu}}$ using (11).
\end{tabular}

\section{B. Downlink Communications}

In this section, an optimal centralized approach is proposed to solve the downlink sum power minimization problem (8). By denoting $\hat{p}_{\mathrm{dtx}}=\sqrt{\tilde{p}_{\mathrm{dtx}}}$, and following similar procedure as in [10], (8) can be equivalently reformulated as the following convex SOCP

$$
\begin{array}{ll}
\text { min. } & q \\
\text { s. t. } & \left\|\frac{\hat{p}_{\mathrm{dtx}} g_{\mathrm{dtx}, \mathrm{cu}}}{\sqrt{N_{0}}}\right\|_{2} \leq \sqrt{\frac{1}{\gamma_{\mathrm{cu}}}} \mathbf{h}_{\mathrm{cu}} \mathbf{m}_{\mathrm{cu}} \\
& \left\|\begin{array}{l}
\mathbf{h}_{\mathrm{drx}} \mathbf{m}_{\mathrm{cu}} \\
\sqrt{N_{0}}
\end{array}\right\|_{2} \leq \sqrt{\frac{1}{\gamma_{\mathrm{drx}}}} \hat{p}_{\mathrm{dtx}} g_{\mathrm{dtx}, \mathrm{drx}} \\
& \left\|\begin{array}{l}
\mathbf{m}_{\mathrm{cu}} \\
\hat{p}_{\mathrm{dtx}}
\end{array}\right\|_{2} \leq q
\end{array}
$$

where the optimization variables are $q, \hat{p}_{\mathrm{dtx}}$, and $\mathbf{m}_{\mathrm{cu}}$. The problem (12) can be solved using standard SOCP solvers via centralized processing if global channel state information (CSI) is available at the BS. As a result, we obtain optimal transmit power for the D2D user and the optimal transmit beamformer for the cellular user.

\section{Practical Considerations}

This section addresses the main (physical layer) implementation aspects of the proposed algorithms, such as the acquirement of global CSI and the required over-the-air signaling for the power control. In the uplink, the proposed approach (i.e., Algorithm 1) requires global CSI to be available at the BS. It is assumed that the BS can measure its local uplink channels, i.e., the channels from the cellular user and the D2D transmitter. In addition, the D2D receiver can also estimate its local channels, i.e., the channels from the D2D transmitter and the cellular user. These scalar channels should be then send to the BS via over-the-air signaling. Having the global CSI, the BS can perform the iterative power control step in Algorithm 1. The resulting optimal uplink powers need to be signaled to the cellular user and D2D transmitter over-the-air. After this, the cellular user and D2D transmitter can use the optimal powers for uplink transmissions. At the BS, the linear MMSE receiver is used for the optimal reception. This power control and beamforming design can be seen as a centralized processing case since the BS is responsible for the power computations relying on the global CSI.

In the downlink phase, the power minimization problem can be solved in a centralized manner. The original problem is reformulated as a convex problem, which can be solved via centralized processing if global CSI is available at the BS. Assuming time-division duplexing (TDD) mode, the BS can acquire its own local downlink channels from the uplink phase due to the channel reciprocity. In addition, the channels from the D2D transmitter to the D2D receiver and to the cellular user need to be delivered to the BS via over-the-air signaling. After this phase, the BS can compute the optimal transmit beamformer for the cellular user and the optimal power for the D2D transmitter. Over-the-air signaling is again used to deliver the power information to the D2D transmitter. Finally, the BS and the D2D transmitter can transmit with optimal parameters. For both uplink and downlink communication phases, in order the obtain the optimal solution and satisfy the rate targets, the global CSI needs to be perfect and all the channels have to be static for the whole process.

\section{Numerical Results}

We consider a single-cell network with a BS serving a single cellular user and an underlaying D2D pair. It is assumed that all the users are located on the cell-edge having fixed distances from the BS. The channel vectors between the BS and users are generated by applying a simple distance dependent pathloss model. The channel between the BS and the cellular user is given by $\mathbf{h}_{\mathrm{cu}}=\sqrt{\left(R / r_{0}\right)^{-\alpha}} \overline{\mathbf{h}}_{\mathrm{cu}}$, where $R$ is the cell radius, $\alpha$ is the pathloss exponent and $r_{0}$ is the far field reference distance. The vector $\overline{\mathbf{h}}_{\mathrm{cu}}$ consists of i.i.d. complex random elements that are generated from the Gaussian distribution, each with zero mean and unit variance. The same model is used to generate the channels from the BS to the D2D transmitter and receiver, i.e., $\mathbf{h}_{\mathrm{dtx}}$ and $\mathbf{h}_{\mathrm{drx}}$, respectively. Moreover, the similar model is also considered for the channels between the cellular and D2D users. Precisely, 


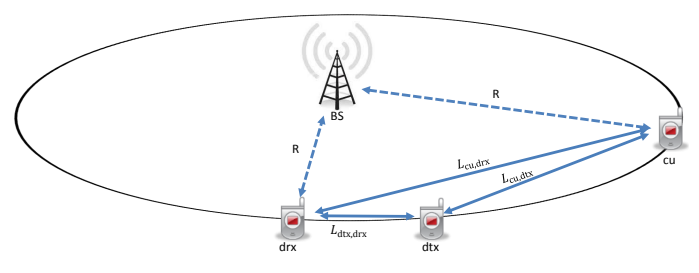

Fig. 2. A single-cell simulation model.

the channel from the D2D transmitter to the D2D receiver is given by $g_{\mathrm{dtx}, \mathrm{drx}}=\sqrt{\left(L_{\mathrm{dtx}, \mathrm{drx}} / l_{0}\right)^{-\alpha}} \bar{g}_{\mathrm{dtx}, \mathrm{drx}}$, where $L_{\mathrm{dtx}, \mathrm{drx}}$ is the distance between the D2D transmitter and receiver, $l_{0}$ is the far field reference distance and $\bar{g}_{\mathrm{dtx}, \mathrm{drx}}$ is the i.i.d. complex scalar channel generated from the Gaussian distribution with zero mean and unit variance. The channels $g_{\mathrm{dtx}, \mathrm{cu}}$ and $g_{\mathrm{cu}, \mathrm{drx}}$ are also generated using the same pathloss model. The simulation model is depicted in Fig. 2.

The sum power performance of the proposed algorithms is compared with the conventional cellular system. In the conventional cellular scenario, the D2D transmitter and receiver are assumed to be regular cellular users which aim to communicate with each other via the BS. Specifically, the other user is transmitting data to the BS in the uplink phase, whereas the other one is receiving data from the BS in the downlink phase. Thus, for a fair performance comparison, the rate target (both in uplink and downlink) of the conventional cellular case (i.e., $R_{\mathrm{c}}$ ) should be twice the rate target of the direct D2D communication case, i.e., $R_{\mathrm{c}}=2 R_{\mathrm{drx}}$. Furthermore, the rate targets are equivalently mapped into the SINR targets as described in section III. For notational simplicity, it is assumed that the resulting SINR targets are the same for the cellular users in the conventional cellular case and for the cellular user in the D2D communication scenario, and this target is denoted by $\gamma$. In all the simulation cases, the average sum power results are achieved by averaging over 1000 channel realizations.

We first study the effect of varying D2D distance on the sum power performance. In this simulation scenario, the distance between the cellular user and D2D receiver is considered constant at $L_{\mathrm{cu}, \mathrm{drx}}=27 \mathrm{~m}$. As illustrated in Fig. 3(a), the locations of the D2D receiver and the cellular user are fixed and only the D2D transmitter is moving towards the cellular user. Fig. 4 shows the sum power versus the D2D distance for OdB SINR targets. The sum power performance of the D2D and conventional cellular systems are compared in uplink and downlink communication phases. The simulation results demonstrate significant improvement in sum power performance of both uplink and downlink underlay in comparison with the conventional cellular system. The performance gain is around $5 \mathrm{~dB}$ for short $\mathrm{D} 2 \mathrm{D}$ distance. However, decreasing the distance between the cellular user and D2D transmitter degrades the performance more rapidly in downlink than in uplink. For this setting, the D2D underlay has no gain when the D2D distance is over 13 meters in the downlink and 22 meters in the uplink. This is due to the fact that in the downlink case, when the source of interference for the cellular user (i.e., the D2D transmitter) is getting closer, the BS needs to use more power to satisfy the SINR target of the cellular user
TABLE I

MAIN SIMULATION PARAMETERS.

\begin{tabular}{|c|c|}
\hline Parameters & Value \\
\hline Cell Radius & $R=400 \mathrm{~m}$ \\
\hline Noise Spectral Density & $N_{0}=-174 \mathrm{dBm}$ \\
\hline Pathloss Exponent & $\alpha=4$ \\
\hline Number of BS Antennas & $T=4$ \\
\hline Number of User's Antennas & 1 \\
\hline Number of Channel Realizations & 1000 \\
\hline Reference Distances & $r_{0}=1 \mathrm{~m}, l_{0}=1 \mathrm{~m}$ \\
\hline
\end{tabular}

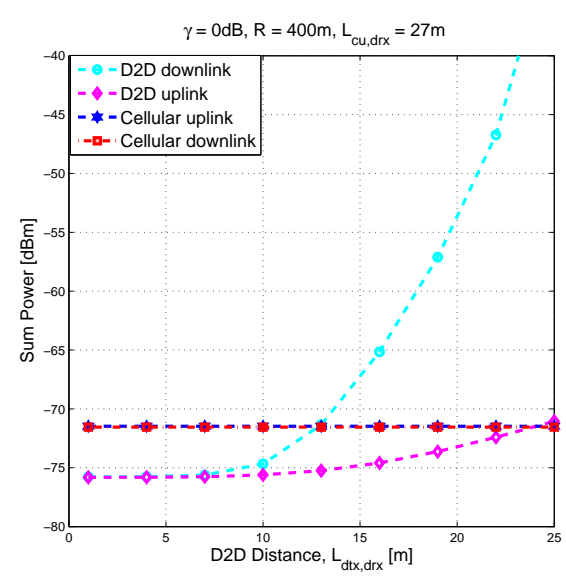

Fig. 4. Sum power versus D2D distance

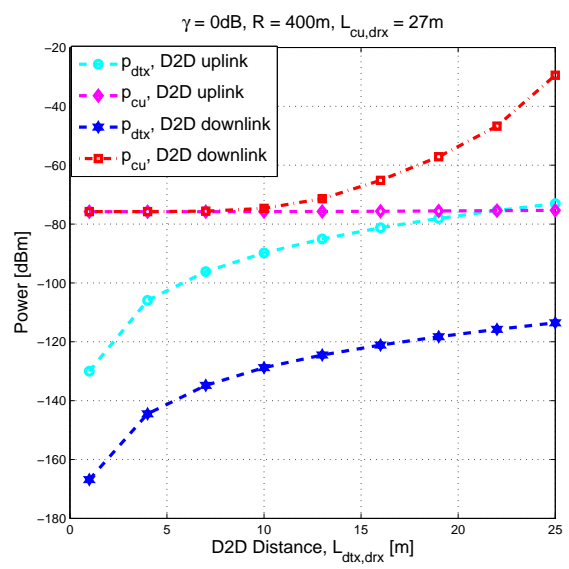

Fig. 5. Individual transmitter powers versus D2D distance. and simultaneously the BS generates more interference to the D2D receiver. Thus, the D2D transmitter needs more power to guarantee the SINR target of the D2D receiver. However, in the uplink case, the distances between the source and the destination of the interferences are remaining the same. Thus, the main reason for the sum power increase is the increase in pathloss due to the longer distance between the D2D transmitter and receiver. Fig. 5 shows the individual transmitter powers versus the D2D distance. The simulation set-up is the same as for Fig. 4, except now only the D2D communication scenario is considered. Results show that the increase of the D2D distance only increases the power of the D2D transmitter. In the downlink phase, the power of the D2D transmitter is increasing faster than that of the BS for short D2D distances (i.e., 1-10 meters). The opposite is happening when the D2D distance is longer (i.e., 13-25 meters).

In the second scenario, we study the effect of distance 


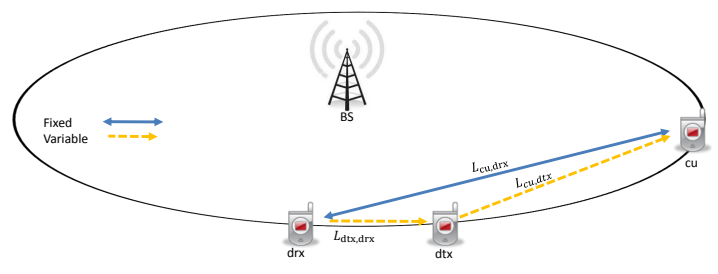

(a) Simulation model for the varying D2D distance.

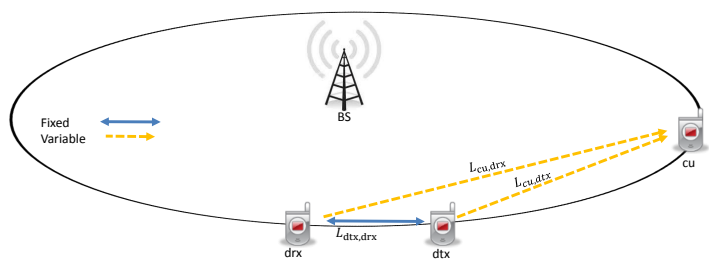

(b) Simulation model for fixed D2D distance while the location of cellular user is varying.

Fig. 3. Simulation models for the first and second scenarios.

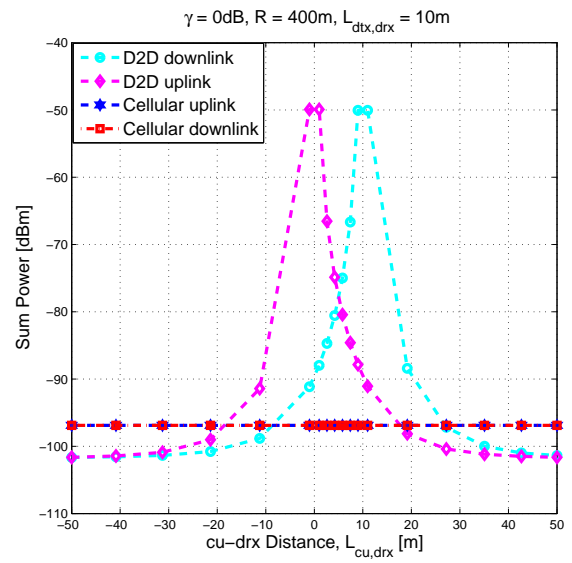

Fig. 6. Sum power versus distance between cellular user and D2D receiver.

between the cellular user and D2D pair on the sum power performance. As depicted in Fig. 3(b), the cellular user moves along the cell circle towards the D2D receiver from a distance of $50 \mathrm{~m}$, crosses over the D2D pair and arrives to a point which is $50 \mathrm{~m}$ away from the D2D receiver in opposite direction. Fig. 6 shows the sum power versus the distance between the cellular user and D2D receiver for $0 \mathrm{~dB}$ SINR targets. The performance of the D2D communication scenario is compared with the conventional cellular system both in uplink and downlink phases. The results show that the locations of the cellular user and D2D pair play important roles for the resource sharing between them. There are four different areas that can be defined from the Fig. 6. First, the cellular user is far enough from the D2D pair. Therefore, the generated interference between them is negligible. Since both uplink and downlink resource sharing are beneficial in this area, it is referred to as open-sharing-area (OSA). Second, the cellular user is close to the D2D receiver. Therefore, the D2D receiver experiences high level of interference, making it a disadvantageous scenario for the uplink resource sharing. Hence, only the downlink resource sharing adds sum power gain to the system. This area is referred to as uplink-exclusivearea (UL-EA). Third, the cellular user is located between the D2D pair. Neither the uplink nor the downlink resource sharing is beneficial in this area. This area is referred to as D2Dexclusive-area (DEA). Fourth, the cellular user is moving away from the D2D transmitter. In this area, the uplink resource sharing adds sum power gain to the system. Nevertheless, the downlink resource sharing is not advantageous because the source of interference for the cellular user (i.e., the D2D transmitter) is close, making it a disadvantageous scenario for the

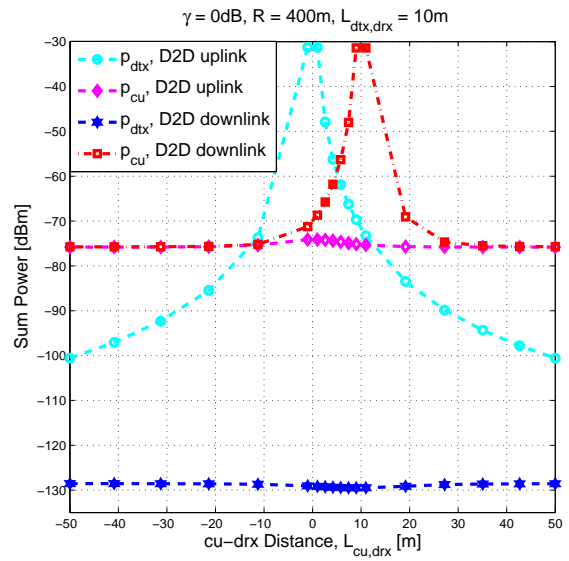

Fig. 7. Individual transmitter powers versus distance between cellular user and $\mathrm{D} 2 \mathrm{D}$ receiver.

TABLE II

CHARACTERISTICS OF FOUR D2D RESOURCE SHARING AREAS.

\begin{tabular}{|c|c|c|c|}
\hline Region & D2D Feasibility & Uplink & Downlink \\
\hline D2D-exclusive-area & $\mathrm{x}$ & $\mathrm{x}$ & $\mathrm{x}$ \\
\hline Uplink-exclusive-area & $\checkmark$ & $\mathrm{x}$ & $\checkmark$ \\
\hline Downlink-exclusive-area & $\checkmark$ & $\checkmark$ & $\mathrm{x}$ \\
\hline Open-sharing-area & $\checkmark$ & $\checkmark$ & $\checkmark$ \\
\hline
\end{tabular}

downlink resource sharing. The aforementioned area is defined as downlink-exclusive-area (DL-EA). Table II summarizes the characteristics of the four D2D resource sharing areas.

Fig. 7 illustrates the individual transmit powers of the cellular user and the D2D transmitter versus the distance between the cellular user and the D2D receiver for uplink and downlink communications. The simulation set-up is the same as Fig. 6, but only the D2D communication scenario is considered herein. Results show that, in the uplink case, the transmission power of the cellular user slightly increases when the cellular user is in DEA, and for the rest of locations it remains approximately fixed. On the contrary, the transmission power of the D2D transmitter is varying significantly while the distance between the cellular user and the D2D receiver is varying. When this distance is short, the source of the interference for the D2D receiver is closer than the desired transmitter. Therefore, the D2D transmitter needs to transmit with higher power level to satisfy the SINR target. In the downlink, the BS is the source of interference towards the D2D receiver. Since the BS is in charge of designing the transmission parameters, this interference can be controlled efficiently due to the transmit beamforming capabilities of the 


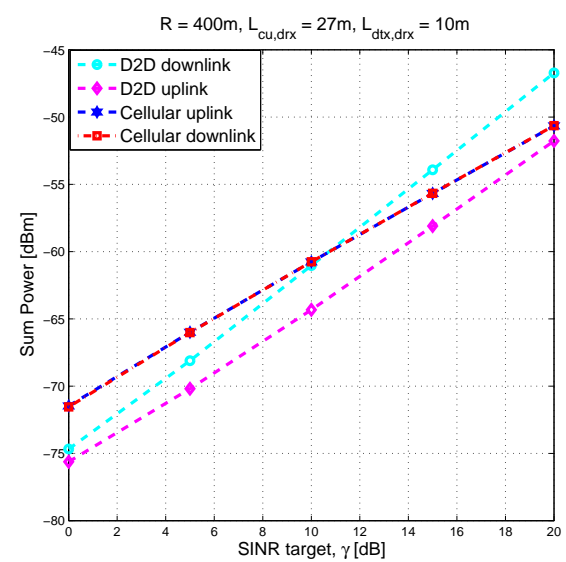

Fig. 8. Sum power versus SINR target.

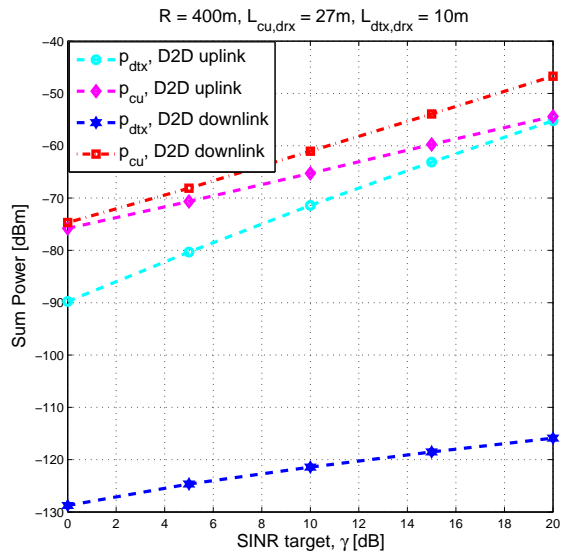

Fig. 9. Individual transmitter powers versus SINR target.

BS. Hence, the transmission power of the D2D transmitter does not vary significantly in UL-EA, DL-EA and OSA, but when the cellular user is located in the DEA, the transmission power of D2D transmitter slightly decreases. This is due to the fact that the transmit beamforming of the BS is close to the zero-forcing (ZF) beamforming. Thus, the generated interference towards the D2D receiver is close to zero. Consequently, the D2D transmitter can transmit with slightly lower power. However, the interference experienced by the cellular user is increasing when the cellular user moves towards the D2D transmitter. Thus, the BS needs to use higher transmission power in order to meet the SINR target of the cellular user.

In the third scenario, we study the effect of the different target SINRs on the achievable power gain. In this simulation setting, the D2D distance is fixed at $10 \mathrm{~m}$ and the distance between the cellular user and D2D receiver is fixed at $27 \mathrm{~m}$. Fig. 8 shows the sum power versus the SINR target. The sum power performance of the underlay D2D and conventional cellular systems are compared in uplink and downlink phases. The results demonstrate that the power gain from the D2D communication decreases when the SINR target increases. Furthermore, for this specific simulation setup, the required sum power in the D2D uplink is lower as compared with the D2D downlink. It can be seen that when the SINR targets increase, the sum power gain decreases for both downlink and uplink, but the degradation of the downlink power gain is faster than the uplink power gain. Hence, for the SINR targets higher than $10 \mathrm{~dB}$, the sum power performance in the downlink phase of the D2D communication case is worse than that in the conventional cellular case. In Fig. 9, the individual transmitter powers are plotted against the SINR target. Results show that the transmit powers of the D2D communication are much lower in comparison with the powers in the cellular communication. Moreover, the used power of the D2D transmitter is significantly lower in the downlink than in the uplink. This again is due to the transmit beamforming performed at the BS.

\section{CONCLUSION}

In this paper, the sum power minimization problem was studied for a cellular system with an underlaying D2D communication. Optimal algorithms were proposed for both uplink and downlink resource sharing cases. The proposed algorithms are benchmarked against the conventional cellular network via numerical examples. The single-cell simulation model consists of a cellular user and a single D2D user pair, which share the same cellular spectrum. The simulation results show that the proposed algorithms can reduce the power consumption of the network by $5 \mathrm{~dB}$ at best as compared with the conventional cellular system. The results also show that the locations of the cellular user and D2D pair play important roles for the resource sharing between them. Based on these results, four resource sharing areas, namely, D2D-exclusive-area, uplink-exclusivearea, downlink-exclusive-area, and open-sharing-area, for D2D communication are defined. As a future work we focus on developing decentralized strategies which are more amenable to practical implementation.

\section{REFERENCES}

[1] H. Min, W. Seo, J. Lee, S. Park, and D. Hong, "Reliability improvement using receive mode selection in the device-to-device uplink period underlaying cellular networks," IEEE Trans. Wireless Commun, vol. 10, no. 2, pp. 413-418, Dec. 2011.

[2] G. Fodor and N. Reider, "A distributed power control scheme for cellular network assisted D2D communications," in Proc. IEEE Global Telecommun. Conf., Houston, TX, 2011, pp. 1-6.

[3] C.-H. Yu, O. Tirkkonen, K. Doppler, and C. Ribeiro, "Power optimization of device-to-device communication underlaying cellular communication," in Proc. IEEE Int. Conf. Commun., Dresden, Germany, 2009.

[4] K. Doppler, M. Rinne, C. Wijting, C. Ribeiro, and K. Hugl, "Deviceto-device communication as an underlay to LTE-advanced networks," IEEE Commun. Mag., vol. 47, no. 12, pp. 42-49, Dec. 2009.

[5] P. Jänis, C.-H. Yu, K. Doppler, C. B. Ribeiro, C. Wijting, K. Hugl, O. Tirkkonen, and V. Koivunen, "Device-to-device communication underlaying cellular communications systems." Int. Journal of Commun., Netw. \& Syst. Sciences, vol. 2, no. 3, pp. 169-178, Mar. 2009.

[6] H. Min, J. Lee, S. Park, and D. Hong, "Capacity enhancement using an interference limited area for device-to-device uplink underlaying cellular networks," IEEE Trans. Wireless Commun., vol. 10, no. 12, pp. 39954000, Dec. 2011

[7] X. Chen, L. Chen, M. Zeng, X. Zhang, and D. Yang, "Downlink resource allocation for device-to-device communication underlaying cellular networks," in Proc. IEEE 23rd Int. Symp. Pers. Indoor and Mobile Radio Commun., Sydney, Australia, 2012, pp. 232-237.

[8] H. Dahrouj and W. Yu, "Coordinated beamforming for the multicell multi-antenna wireless system," IEEE Trans. Wireless Commun, vol. 9, no. 5, pp. 1748-1759, May. 2010.

[9] M. Chiang, P. Hande, T. Lan, and C. W. Tan, "Power control in wireless cellular networks," Found. Trends Netw., vol. 2, no. 4, pp. 381-533, Apr. 2008. [Online]. Available: http://dx.doi.org/10.1561/1300000009

[10] A. Wiesel, Y. C. Eldar, and S. Shamai, "Linear precoding via conic optimization for fixed MIMO receivers," IEEE Trans. Signal Process., vol. 54, no. 1, pp. 161-176, Jan. 2006. 